\title{
Adesão e dificuldades relacionadas ao tratamento medicamentoso em pacientes com depressão
}

\author{
Adherence and difficulties related to drug treatment in patients with depression
}

Adhesión y dificultades relacionadas al tratamiento medicamentoso en pacientes con depresión

\section{Grazielle Ibanez', Bruna Paiva do Carmo Mercedes', Kelly Graziani Giacchero Vedana', Adriana Inocenti Miasso'}

\author{
'Universidade de São Paulo, Escola de Enfermagem de Ribeirão Preto, \\ Departamento de Enfermagem Psiquiátrica e Ciências Humanas. Ribeirão Preto-SP, Brasil.
}

Submissão: 29-10-2012 Aprovação: 30-06-2014

\section{RESUMO}

Esta pesquisa verificou a adesão e conhecimento de pessoas com depressão quanto à farmacoterapia prescrita, a satisfação com a equipe de saúde e as dificuldades relacionadas ao tratamento medicamentoso. Trata-se de estudo transversal, descritivo, com abordagem quali-quantitativa. Participaram do estudo 27 pessoas atendidas em um serviço ambulatorial do interior de São Paulo - Brasil. Os dados foram coletados por entrevistas semiestruturadas e revisão de prontuários e analisados por estatística descritiva e análise de conteúdo. Constatou-se que 29,6\% dos pacientes não aderiam aos medicamentos e que 51,9\% desconheciam a dose dos medicamentos prescritos. Como dificuldades para o seguimento da terapêutica medicamentosa, foram apontados o impacto dos sintomas depressivos sobre o autocuidado, a insatisfação com efeitos dos psicofármacos, a carência de apoio, a polifarmacoterapia e a falta de conhecimento sobre o transtorno e tratamento. Este estudo aponta fatores críticos para a promoção da segurança do paciente no seguimento da terapêutica medicamentosa.

Descritores: Transtorno Depressivo; Antidepressivos; Adesão à Medicação. with the health team and difficulties related to drug treatment. This is descriptive and cross-sectional study, with quail-quantitative approach. The study included 27 people assisted in an outpatient clinic in the interior of the state of São Paulo - Brazil. Data were collected through semi-structured interviews and review of medical records and analyzed using descriptive statistics and content analysis. It was found that $29.6 \%$ of patients were no adherent to medication and $51.9 \%$ were unaware of the dose of prescribed drugs. Impact of drug therapy in depressive symptoms on self-care, dissatisfaction with effects of psychoactive drugs, lack of support, use of multiple medications and lack of knowledge about the disorder and treatment were reported as difficulties in following drug therapy. This study shows critical factors to the promotion of patient safety in following drug therapy.

Key words: Depressive Disorder; Antidepressive Agents; Medication Adherence.

\section{RESUMEN}

Esta investigación verificó la adhesión y el conocimiento de personas con depresión cuanto al tratamiento farmacológico prescrito, la satisfacción con el equipo de salud y las dificultades relacionadas al tratamiento medicamentoso. Estudio transversal, descriptivo, con enfoque cuali-cuantitativo. Participaron 27 personas asistidas en una clínica ambulatoria en el interior de São Paulo - Brasil. Datos fueron recolectados a través de entrevistas semi-estructuradas y revisión de registros médicos y analizados por estadística descriptiva y análisis de contenido. Se encontró 29,6\% de los pacientes no adherían a medicación y 51,9\% no tenían conocimiento de la dosis de medicamentos prescritos. Como dificultades para el seguimiento del tratamiento medicamentoso, fueron citados el impacto de los síntomas depresivos en el autocuidado, insatisfacción con los efectos de psicofármacos, falta de apoyo, uso de múltiples medicamentos y falta de conocimiento sobre la enfermedad y tratamiento. Este estudio apunta factores críticos para promoción de la seguridad del paciente en seguimiento del tratamiento medicamentoso.

Palabras clave: Trastorno Depresivo; Antidepresivos; Cumplimiento de la Medicación.

\section{AUTOR CORRESPONDENTE Adriana Inocenti Miasso E-mail: amiasso@eerp.usp.br}




\section{INTRODUÇÃO}

A depressão é um transtorno mental comumente crônico associado à incapacitação, ao sofrimento psíquico ${ }^{(1)}$ e físico ${ }^{(2)}$ e a sobrecarga familiar ${ }^{(3)}$. Sua prevalência anual na população em geral varia de $3 \%$ a $11 \%{ }^{(1)}$.

A depender da gravidade, esse transtorno requer tratamento medicamentoso para o controle dos sintomas. O tratamento medicamentoso da depressão precisa ser continuado por tempo variável após a remissão dos sintomas ${ }^{(1)}$. O seguimento adequado da prescrição medicamentosa é necessário para obtenção de benefícios clínicos e redução dos riscos de recaída e recorrência. No entanto, a maior parte dos pacientes que recebe prescrição de antidepressivos interrompe prematuramente o tratamento ou o conduz de forma inconsistente ${ }^{(4)}$. A não adesão ao tratamento destaca-se como fator potencialmente modificável que pode precipitar recaídas ${ }^{(5)}$ e refratariedade farmacológica ${ }^{(6)}$.

Existe uma ampla variedade de fatores que contribuem para o uso inadequado dos medicamentos ${ }^{(4,7)}$. Essas barreiras à adesão ao medicamento precisam ser antecipadas e exploradas cuidadosamente durante o tratamento ${ }^{(4)}$, pois a participação do paciente é determinante no processo do tratamento.

Desse modo, é importante conhecer os fatores envolvidos no seguimento da terapêutica medicamentosa pela pessoa com depressão, para implementação de ações que melhorem a adesão e contribuam para prevenção de agravos decorrentes do uso inadequado de medicamentos.

Nessa perspectiva, este estudo teve como objetivos verificar a adesão e conhecimento de pessoas com depressão quanto à farmacoterapia prescrita; identificar as dificuldades relacionadas ao seguimento da terapêutica medicamentosa e avaliar a satisfação da pessoa com depressão acerca da equipe de saúde que a assiste e da terapêutica medicamentosa instituída.

\section{METODOLOGIA}

Trata-se de um estudo transversal, descritivo, com abordagem quali-quantitativa. A pesquisa foi realizada em um Núcleo de Saúde Mental (NSM) pertencente ao Sistema Público de Saúde e localizado no interior de São Paulo, Brasil. O projeto foi desenvolvido após aprovação por Comitê de Ética em Pesquisa (Protocolo no 380/CEP-CSE-FMRP-USP).

Foram elegíveis para o estudo todos os pacientes que tiveram consulta médica agendada no local do estudo no período de $1^{\circ}$ de agosto a 31 de outubro de 2011 e que preencheram os seguintes critérios de inclusão: ter diagnóstico de depressão unipolar (estabelecido pelo médico psiquiatra do local de estudo) e ter prescrição de uso contínuo de medicamentos para tratamento da depressão. Foram excluídos da amostra os pacientes com menos de 18 anos de idade, incapazes de se comunicar verbalmente em português e sem atualização do número de telefone ou do endereço no prontuário.

Para a coleta dos dados foram realizadas a revisão de prontuários e entrevista semiestruturada gravada guiadas por um roteiro elaborado pelas autoras do estudo contendo questões sobre dados sociodemográficos e tratamento dos pacientes, um teste que avalia a adesão do indivíduo à farmacoterapia ${ }^{(8)}$ e uma escala que permite avaliar o conhecimento do entrevistado sobre a terapêutica medicamentosa prescrita(9).

O grau de adesão foi definido pela aplicação do Teste de Medida de Adesão (MAT) ${ }^{(8)}$. Este teste é composto por sete questões. Para cada questão, seguem-se as respostas do tipo Likert. Após a obtenção dos dados, os valores correspondentes às respostas de cada questão do MAT são somados e divididos pelo número total de questões. O valor encontrado após esse procedimento é convertido numa escala dicotômica para identificar os pacientes que apresentam adesão ou não adesão ao tratamento medicamentoso.

Para identificar o grau de conhecimento do paciente, sobre os medicamentos prescritos, foi adotada escala já empregada em estudos anteriores ${ }^{(9)}$. O referido instrumento, indica como traduzir, para porcentagens, a quantidade (números) de informações que o paciente possui e direciona a categorização desse conhecimento.

Esse instrumento pressupõe que o grau de conhecimento de uma pessoa sobre cada aspecto relacionado às medicações (nome, dose e frequência de utilização) pode ser pontuado de 0 a $100 \%$ e classificado em intervalos regulares, que representam as seguintes classes: sem conhecimento $(0 \%)$; muito pouco conhecimento $(0 \%-\mid \% 25 \%)$; pouco conhecimento $(25 \%-\mid \% 50 \%)$; conhecimento regular $(50 \%-\mid \% 75 \%)$ e bom conhecimento $(75 \%-\mid \% 100 \%)$.

As respostas dos pacientes foram comparadas com os dados contidos no prontuário. A resposta a cada pergunta foi classificada como certa ou errada, considerando-se os itens avaliados para cada um dos medicamentos prescritos. A resposta "não sei" foi classificada como errada. Assim, se fossem prescritas 10 medicações para uma pessoa que soubesse informar corretamente o nome de quatro delas, seu grau de conhecimento sobre os nomes dos fármacos seria de $40 \%$ e estaria incluído na categoria "pouco conhecimento" que corresponde ao intervalo de pontuação $25 \%-\mid \% 50 \%$. Procedeu-se dessa forma para cada variável relacionada aos medicamentos prescritos.

Para análise dos dados relacionados à caracterização dos pacientes, conhecimento e adesão ao medicamento, foi utilizada a estatística descritiva. Para análise dos dados qualitativos, foi adotada a análise de conteúdo ${ }^{(10)}$. Primeiramente, os dados foram reunidos e organizados. Por meio da análise do material foram estabelecidas categorias empíricas. Posteriormente, foi realizada articulação do material empírico com a literatura.

Para preservar o anonimato dos participantes do estudo, os entrevistados foram identificados, em seus depoimentos, com a letra "S" acrescida do número que corresponde à ordem de realização das entrevistas.

\section{RESULTADOS}

Participaram do estudo 27 pessoas. Dentre os entrevistados, $24(88,9 \%)$ eram do sexo feminino, $17(63,0 \%)$ tinham de 50 a 69 anos de idade, $18(66,7 \%)$ eram casados, 26 
(96,3\%) não possuíam vínculo empregatício, 16 (59,3\%) não haviam completado o ensino fundamental e 16 (59,3\%) possuíam renda familiar de um a três salários mínimos.

\section{Adesão e conhecimento relacionados à farmacoterapia prescrita}

De acordo com a avaliação da adesão realizada por meio do teste MAT, 29,6\% dos pacientes não aderiam ao tratamento.

Esta pesquisa também avaliou o grau de conhecimento do paciente em relação aos medicamentos prescritos. Para tanto, a última prescrição médica disponível no prontuário do paciente foi comparada aos medicamentos que o paciente referiu utilizar. Desse modo, foi analisado o grau de conhecimento referente ao nome, dose e frequência de administração dos medicamentos, conforme demonstra o Gráfico 1.

Gráfico 1 - Distribuição dos sujeitos do estudo de acordo com o grau de conhecimento sobre o nome, frequência e dose dos medicamentos prescritos

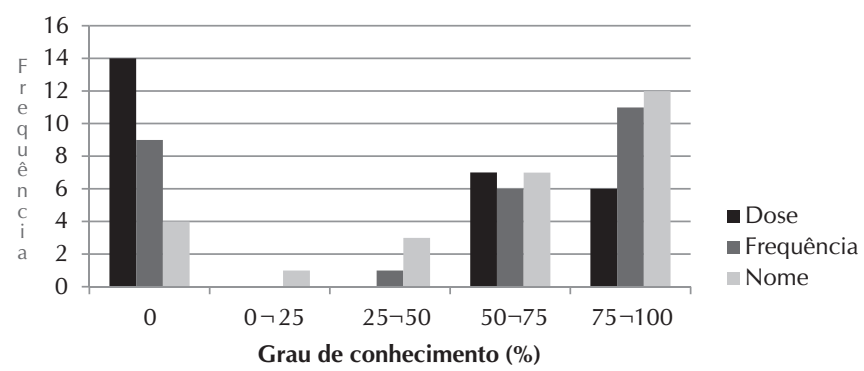

Foi possível perceber que o menor grau de conhecimento foi em relação à dose e à frequência de administração dos medicamentos. Respectivamente, $51,9 \%$ e $33,4 \%$ dos pacientes apresentaram $0 \%$ de conhecimento a respeito de tais aspectos da terapêutica prescrita. Na avaliação das informações referidas sobre a dose dos fármacos, foram consideradas corretas as respostas que identificavam a quantidade, em unidades de medida (grama, miligrama e mililitro), a ser administrada em cada horário ou no período de 24 horas, de acordo com a prescrição médica.

Observa-se ainda, no Gráfico 1, que 44,5\% dos pacientes sabiam informar mais de $75 \%$ dos nomes dos medicamentos em uso. Para avaliação do conhecimento quanto aos nomes dos medicamentos, aceitou-se como correta a resposta que identificasse o medicamento prescrito, tanto pelo nome genérico como por qualquer nome comercial.

\section{Dificuldades relacionadas ao seguimento da terapêutica medicamentosa}

A análise dos dados qualitativos relacionados às dificuldades enfrentadas pelos pacientes no seguimento da terapêutica medicamentosa resultou na construção das seguintes categorias: "Os sintomas da depressão", "Efeitos limitados e desgastantes da medicação e a polifarmacoterapia", "A necessidade de apoio" e "Pouco conhecimento sobre a depressão e o tratamento".

\section{Os sintomas da depressão}

A sintomatologia da depressão, especialmente quando aliada aos estressores do cotidiano, foi uma dificuldade enfrentada pelos pacientes no seguimento da terapêutica medicamentosa. O paciente deprimido pode experimentar desesperança, avolição, falta de energia e iniciativa, lentidão, falta de concentração, desejo de morrer, menor sensação de autocontrole e dificuldade para tomar decisões.

Falta de motivo para viver. Não tem ânimo, você não tem coragem de fazer nada [...] o corpo não obedece. É como se a cabeça não tivesse mais autoridade sobre mim [...] é como se eu tivesse em uma cadeira de rodas. Um deficiente, ele é obrigado a estar na cadeira de roda. O depressivo não! Ele não pode ver uma cadeira de roda que ele senta e fica e não tem vontade de levantar [...] eu não tinha força para levantar um braço, eu não andava [...] a depressão, eu acho que é isso. Ela me irrita tanto, é um inimigo que eu não consigo ver. E ela me derruba, ela me prejudica, ela me faz mal. (S9)

Por que eu já tive depressão de cair de cama de não comer e ficar sem comer e ficar fraquinha [...] amoleceu meus nervos todos. Meu marido precisou sair de férias, para me ajudar a ir ao banheiro. (S11)

Os sintomas da depressão podem comprometer o desempenho do paciente em diversas atividades e, consequentemente, para a autoadministração dos medicamentos prescritos.

Se meu estômago fica ruim aí, eu não consigo (tomar o medicamento). Porque se eu tomar, eu vou vomitar, mas não por causa dele (medicamento), por causa dos sintomas. Porque quando eu fico nervosa, aí já não sinto vontade de comer. (S10)

O paciente sintomático pode ficar mais propenso a não aderir à prescrição medicamentosa tanto interrompendo o uso de fármacos prescritos quanto ingerindo medicações sem prescrição. A medicação pode ser utilizada, inclusive, como um instrumento para tentativas de suicídio.

Eu não comia, não dormia, eu chorava muito. Às vezes me dava aquele estado de nervoso e eu começava a ter vontade de quebrar tudo, eu tomava muito remédio, porque aí, eu ia aos remédios [...]. Várias vezes eu tive que ir para lá (hospital) fiquei várias vezes internada lá para poder controlar a medicação que eu tomava em excesso. (S19)

A pessoa com depressão vivencia o impacto do transtorno em seu cotidiano e tem, no medicamento, uma oportunidade para atenuar os sintomas depressivos e suas consequências. Todavia, o paciente percebe que o tratamento farmacológico possui fragilidades e também lhe proporciona experiências negativas.

\section{Efeitos limitados e desgastantes da medicação e a polifar- macoterapia}

Os pacientes expressaram ambivalência em relação à terapêutica medicamentosa. Embora fosse necessário e útil para 
aliviar os sintomas, o tratamento medicamentoso contínuo era desgastante e nem sempre proporcionava resultados condizentes com as expectativas dos pacientes.

Eu tenho que conviver com eles (medicamentos), então é bom. É horrível, mas ao mesmo tempo acaba sendo bom tomar o remédio porque aí a gente melhora. (S23)

Em alguns momentos, os pacientes experimentavam insatisfação com os efeitos do tratamento farmacológico por considerá-los limitados e desgastantes. A ação dos medicamentos foi considerada limitada quando não era suficiente para eliminar ou reduzir, de forma acentuada, os sintomas da depressão.

Faz tanto tempo que eu tomo ele (medicamento) que eu acho que nem efeito não faz mais [...] Parece que começo dá uma melhorada assim, só que a gente fica assim chapada. É como se tivesse drogado. (S8)

Ao invés de eu melhorar, eu ficava muito pior. Mas os remédios que me davam, me deixavam mais deprimida. Então em vez de eu melhorar, parece que o negócio piorava cada vez mais. (S21)

De acordo com os depoimentos, o seguimento da terapêutica medicamentosa era considerado desgastante por diversos motivos, entre os quais se destacaram os efeitos colaterais, a necessidade de abster-se de outras substâncias psicoativas e a longa duração do tratamento.

Eu acho difícil só que eu não deixo de tomar. Eu falo: oh, meu Deus! Mas será até quando, que eu vou tomar remédio desse jeito. Meu estômago já está ruim, não está aguentando mais remédio. Mas estou tomando. (S6)

Eu só queria que tivesse um ponto final, só que eu só to vendo "reticências". (S9)

Será que eu sempre vou tomar para o resto da minha vida? Será que essa depressão nunca vai sair de dentro de mim? (S22)

A necessidade de ingerir vários tipos de medicamentos ou grande quantidade de comprimidos diariamente foi apontada como uma dificuldade no cotidiano dos pacientes. Além dos medicamentos prescritos para o controle da depressão, algumas pessoas utilizavam medicamentos para o tratamento de comorbidades.

Eu tomo muita medicação. Eu tomo 14 comprimidos por dia, porque eu tenho problema no coração, pressão, eu tenho diabete, eu tenho o colesterol. (S7)

Nesse contexto, houve pacientes que se queixavam de precisar de muitas medicações por acreditar que a prescrição de vários medicamentos sinaliza um agravo à condição de saúde do indivíduo.
É eu acho chato, ver a mão assim cheia de remédio, falo: "que situação que eu cheguei". (S15)

Os sintomas do transtorno, aliados às dificuldades experimentadas no cotidiano e à ambivalência em relação aos medicamentos fazia com que os pacientes identificassem a necessidade de suporte, especialmente das pessoas que Ihes eram significativas.

\section{A necessidade de apoio}

Os sintomas decorrentes da depressão faziam com que, em algumas situações, o paciente se sentisse vulnerável e com necessidade de apoio em diferentes esferas de sua vida, inclusive em relação ao tratamento.

Tanto do remédio quanto da comida, ela (familiar) pega no pé. Acho, eu acho que isso aí é o carinho que ela tem por mim e está me olhando, me ajudando, é isso. Ela deixa os horários e as medicações todas separadas em cima da mesa. (S6)

Sem a ajuda de alguém, não faz nada! Eu acho muito importante ter as minhas filhas ao meu lado, meu filho. Estão sempre assim: "Oh mãe, a senhora já tomou o remédio?" Eu falo: "já". Às vezes: "Não, eu não tomei não". (Os filhos dizem):

"Então toma agora antes que a senhora se esqueça". (S5)

Todavia, nem sempre pessoas significativas para o paciente, como familiares e amigos Ihe ofereciam auxílio e incentivo para a manutenção da terapêutica medicamentosa.

Eu não tenho ajuda assim em casa, ninguém entende o que é a depressão. [...] (O medicamento) era para estar nas mãos dos meus filhos, do meu marido, mais fica na minha mão [...] então, quer dizer, se der um "curto" aqui, me der vontade de tomar tudo aquilo lá que está parado eu tomo [...] esses dias eu fiquei muito agitada, nervosa eu pensei em tomar tudo, e na hora que eu tiver apagando, meter fogo na cama. (S1)

Além disso, o paciente podia ser desencorajado a seguir a prescrição medicamentosa. Crenças conflitantes com o saber médico, a negação do acometimento do paciente pela depressão e o desconhecimento sobre a seriedade e evolução do transtorno podem favorecer que familiares e amigos considerem o tratamento medicamentoso desnecessário. Desse modo, esses indivíduos podem incentivar o paciente a descontinuar o tratamento.

Os meus familiares acham que eu não devo tomar (o medicamento), porque vou ficar dependente [...] então é muito difícil! (S3)

Além das dificuldades relacionadas aos sintomas depressivos, aos efeitos dos medicamentos e à carência de suporte, os pacientes experimentavam dificuldades decorrentes do conhecimento insuficiente sobre o transtorno e tratamento. 


\section{Pouco conhecimento sobre a depressão e o tratamento}

A falta de conhecimento sobre a depressão e o tratamento é um fator que pode comprometer a segurança do paciente no seguimento da terapêutica medicamentosa. Entre os participantes do estudo, houve aqueles que desconheciam o nome, posologia, efeitos terapêuticos e colaterais e cuidados relacionados aos fármacos.

Depressão eu sei por que eu vi um dia o médico dando entrevista na televisão que é uma parte que a gente tem assim que não produz lá o hormônio. [...] (O medicamento) eu sei que é para depressão, agora o efeito que ele faz eu não sei. (S13)

Tenho minhas dúvidas ainda. (S18)

O déficit de conhecimento foi atribuído à falta de transmissão de conhecimentos pela equipe de saúde, esquecimento ou incompreensão de informações fornecidas. Desse modo, alguns pacientes buscavam informações sobre os medicamentos em fontes nem sempre seguras, tais como jornais, revistas, internet, programas de televisão, bulas e pessoas conhecidas.

Eu não sei falar muito bem os nomes dos medicamentos que eu tomo [...] Sabe o que eu estou tomando agora? Esse tal de AAS de criança. [...] Sem indicação. O médico estava falando na televisão que é bom. Aí, eu pensei: então acho que eu vou beber também. (S10)

Destaca-se que o enfermeiro não foi identificado como um colaborador na educação do paciente, no serviço estudado.

Eu nunca conversei com o enfermeiro não. Só o atendimento lá na portaria quando eu chego o moço atende, pega o cartão e eu passo para a médica. Eu nunca passei com enfermeira não. (S3)

O presente estudo teve ainda como objetivo avaliar a satisfação da pessoa com depressão acerca da equipe de saúde e do tratamento medicamentoso. A satisfação com a terapêutica medicamentosa oscila ao longo da trajetória do paciente, pois ele é ambivalente em relação à farmacoterapia, como já descrito na categoria "Efeitos limitados e desgastantes da medicação e a polifarmacoterapia". O tópico seguinte apresenta a satisfação da pessoa com depressão com a equipe de saúde que a assiste.

\section{Satisfação com a equipe de saúde: a gratidão pelo trata-} mento "gratuito"

A maior parte dos pacientes referiu satisfação com a equipe de saúde. Os entrevistados consideravam injusto criticar a equipe de saúde e tinham gratidão pelo tratamento que lhes era fornecido "gratuitamente".

A possibilidade de ser atendido em situações críticas e de obter melhora dos sintomas foi citada como um motivo para ser grato ao trabalhador da saúde. Também esteve presente nos depoimentos a sensação de ser afortunado por ter acesso ao serviço de saúde e o temor de "perder a vaga" no referido serviço.
Eu não posso fala que é mal. É muito bom para mim, foi ótimo. Foi melhor que se eu tivesse dinheiro e pagasse um médico particular. (S7)

Todavia, mesmo em relatos de pacientes que se declararam satisfeitos foram apontadas falhas no atendimento. As queixas mais frequentes estiveram relacionadas à falta de escuta e de orientações pelos profissionais, longa espera para obter acesso ao atendimento e intervalo de tempo prolongado entre as consultas.

Não posso reclamar de ninguém aqui [...]. Eu tenho medo de perder aqui a minha vaga. [...] É muita gente para os médicos, então eles não tem tempo para a gente, assim, para falar tudo. (S1)

Eu acho que se tivesse uma enfermeira ou mesmo o médico que explicasse para o paciente... Porque geralmente vem, ele olha a pasta e pergunta "está tudo bem?" E ai já prescreve o remédio de novo, não faz assim uma pergunta, "como que você passou?" "Teve algum problema?". (S2)

Mas eu acho que o espaço é muito longo. Dos retornos. Eu fiquei um ano, ano passado eu não vim nenhuma vez. (S22)

Desse modo, embora a maioria dos entrevistados tenha relatado estar satisfeita com o atendimento oferecido, os argumentos insuficientes e as queixas fazem com que a afirmação de satisfação pareça inconsistente.

\section{DISCUSSÃO}

Este estudo investigou 27 pessoas com depressão unipolar e observou que $29,6 \%$ dos participantes não aderiam ao tratamento medicamentoso. Os resultados do presente estudo são contrastantes com a literatura, pois, a depressão está associada à baixa adesão ao regime terapêutico ${ }^{(11)}$ e estudos relevam que aproximadamente metade dos pacientes interrompe o tratamento com antidepressivos nos primeiros seis meses de tratamento ${ }^{(4,12-13)}$. Desse modo, a adesão entre pacientes depressivos é considerada importante desafio para o sucesso do tratamento farmacológico na prática clínica ${ }^{(4)}$.

O transtorno depressivo maior pode se cronificar e está associado a níveis altos de incapacitação funcional(1), à má evolução de doenças clínicas concomitantes ${ }^{(2)}$ e ao suicídio ${ }^{(14)}$. Os resultados deste estudo evidenciaram que pacientes sintomáticos podem ter comprometimento para o autocuidado e manutenção do tratamento medicamentoso, podendo, inclusive, utilizar os medicamentos para cometer suicídio. Desse modo, o paciente sintomático requer atenção especial em relação ao uso de medicamentos, pois, caso os interrompa ou utilize de forma inadequada, pode ter os sintomas intensificados, iniciando um possível ciclo vicioso.

Embora se percebessem vulneráveis e com necessidade de apoio, nem sempre os pacientes recebiam auxílio e incentivo para a manutenção do tratamento farmacológico. Houve participantes desencorajados a seguir a prescrição medicamentosa por pessoas que consideravam o tratamento desnecessário. 
A inclusão da família no tratamento de pessoas com depressão comumente é desejável pelos pacientes e está associada a melhores resultados no tratamento ${ }^{(15)}$.

Outra dificuldade apontada pelos pacientes foi a ambivalência destes em relação à terapêutica medicamentosa. Apesar dos resultados positivos, antidepressivos apresentam limitações, como o tempo de latência para o início do efeito sobre sintomas subjetivos da depressão e a presença de efeitos colaterais. Além disso, aproximadamente $50 \%$ dos casos de depressão não respondem satisfatoriamente ao tratamento de primeira escolha realizado de modo adequado. Essas limitações podem contribuir para o abandono do tratamento com antidepressivos ${ }^{(7)}$.

A polifarmacoterapia foi outra dificuldade apontada pelos pacientes. Além de simbolizar um possível agravo à condição de saúde do indivíduo, a necessidade de ingerir muitos medicamentos pode dificultar o conhecimento e seguimento do esquema terapêutico e elevar a probabilidade de efeitos colaterais ${ }^{(16)}$.

Nesta pesquisa também foi analisado o conhecimento do paciente sobre o esquema terapêutico, que representa condição básica para a autoadministração dos fármacos prescritos. O maior índice de desconhecimento foi quanto à dose, seguida pela frequencia de administração dos fármacos. A falta dessas informações pode dificultar a manutenção do fármaco em doses terapêuticas, comprometendo a eficácia e segurança do tratamento medicamentoso.

Neste estudo, o déficit de conhecimento foi atribuído à falta de transmissão de conhecimentos pela equipe de saúde, esquecimento ou incompreensão de informações recebidas. Deste modo, evidencia-se a necessidade de maior qualificação, investimento e avaliação de estratégias psicoeducativas.

Um agravante a essa situação é o fato de que familiares de pessoas com transtornos mentais também possuem déficit de conhecimento sobre os medicamentos prescritos para o paciente, o que limita sua possibilidade de intervenção para auxiliar o paciente a manter o medicamento em níveis terapêuticos ${ }^{(17)}$. Nesse contexto, destaca-se a carência de apoio e informações fornecidas a esses familiares nos serviços de saúde ${ }^{(18)}$.

O presente estudo investigou ainda a satisfação dos pacientes sobre a equipe de saúde que o assiste. A maior parte dos participantes se declarou satisfeita com a equipe de saúde. Todavia, essa satisfação esteve atrelada à gratidão pela "gratuidade" do tratamento e à sensação de ser afortunado por obter acesso ao serviço. Entre as insatisfações dos pacientes predominaram a falta de escuta e de orientações pelos profissionais, a dificuldade de acesso ao atendimento e o período prolongado entre as consultas.

O tratamento "gratuito" foi apreciado como uma dádiva ou favor alcançado sem merecimento e não como um direito dos cidadãos. Tal percepção do tratamento disponibilizado na rede pública pode propiciar a postura passiva e conformada do usuário perante a equipe de saúde.

A literatura aponta que, embora os usuários avaliem positivamente os serviços de saúde públicos, apresentam queixas relacionadas à demora em obter atendimento, falta de humanização e acolhimento, bem como déficit de recursos físicos e materiais $^{(19)}$. Todavia, com a implantação do Sistema Único de Saúde (SUS), teoricamente, qualquer pessoa tem direito à assistência de qualidade. A Enfermagem pode contribuir significativamente com os usuários de serviços públicos orientando-os sobre seus direitos de cidadãos à assistência em saúde qualificada.

\section{CONSIDERAÇÕES FINAIS}

Este estudo investigou a adesão e conhecimento de pessoas com depressão quanto à terapêutica medicamentosa prescrita, identificou as dificuldades relacionadas ao seguimento da farmacoterapia e avaliou a satisfação dos pacientes com a equipe de saúde.

Verificou-se baixa taxa de não adesão entre os participantes do estudo, comparativamente à literatura descrita. Contudo, grande parcela dos pacientes desconhecia parte das informações relacionadas ao esquema terapêutico. Desse modo, embora aderentes ao tratamento, os participantes do estudo, podem ter comprometimento na segurança no seguimento da farmacoterapia, pois para utilizar adequadamente e de forma segura os medicamentos prescritos, subentende-se que seja necessário conhecer a prescrição.

Além do esquema terapêutico, o paciente precisa conhecer outros elementos relacionados ao transtorno, tratamento, manejo de sintomas, estilo de vida saudável, entre outros. A educação em saúde é uma importante intervenção de enfermagem que não foi evidente no serviço de saúde estudado.

Enfermeiros devem ser devidamente preparados, conscientizados e motivados para exercer a educação em saúde de forma atualizada, efetiva e individualizada, considerando as necessidades, limitações, potencialidades e interesses do paciente.

A satisfação com a equipe de saúde foi justificada pela gratidão pelo acesso e "gratuidade" do tratamento e não pela avaliação crítica sobre a assistência. Tal achado revela a importância de ações intersetoriais que objetivem elevar a conscientização de pessoas com transtornos mentais e da sociedade em geral sobre seus direitos e deveres como cidadãos.

Ao falar sobre as dificuldades enfrentadas no seguimento da terapêutica medicamentosa, os participantes do estudo apontaram as seguintes questões: o impacto dos sintomas da depressão sobre a capacidade para o autocuidado, a insatisfação com os efeitos da farmacoterapia, a vulnerabilidade do paciente e necessidade de apoio (que nem sempre é disponibilizado), a polifarmacoterapia e a falta de conhecimento sobre o transtorno e o tratamento.

Essas dificuldades vivenciadas pelos pacientes com depressão revelam a importância do profissional de enfermagem atentar-se para a identificação desses problemas em outros contextos. A Enfermagem tem papel fundamental na escolha de intervenções apropriadas para minimizar efeitos colaterais e incapacidades, para promover o conhecimento e habilidades para enfrentamento adaptativo do transtorno e para incluir a família como alvo e parceira no cuidado.

O presente estudo traz importantes contribuições para a prática clínica e pesquisa em saúde mental ao apontar fatores que podem prejudicar a segurança do paciente com diagnóstico de depressão no seguimento da terapêutica medicamentosa. Destaca-se a importância de estudos que proponham e avaliem estratégias voltadas para a educação, supervisão e motivação para a adesão, bem como, para a inclusão de familiares na assistência. 


\section{REFERÊNCIAS}

1. Fleck MP, Berlim MT, Lafer B, Sougey EB, Del Porto JA, Brasil MA, et al. Revisão das diretrizes da Associação Médica Brasileira para o tratamento da depressão (versão integral). Rev Bras Psiquiatr [Internet]. 2009 [acesso em 29 de outubro de 2012];31(Suppl.1):7-17. Disponível em: http://www.scielo.br/scielo.php?pid = S1516-444620 09000500003\&script $=$ sci_arttext

2. Bivanco-Lima D, Santos IS, Vannucchi AMC, Ribeiro MCSA. Cardiovascular risk in individuals with depression. Rev Assoc Med Bras [Internet]. 2013 [cited 2012 october 29];59(3):298-304. Available from: http://www. ncbi.nlm.nih.gov/pubmed/23684214

3. Koujalgi SR, Patil SR. Family burden in patient with schizophrenia and depressive disorder: a comparative study. Indian J Psychol Med [Internet]. 2013 [cited 2012 october 29];35(3):251-5. Available from: http://www. ncbi.nlm.nih.gov/pubmed/24249926

4. Sansone RA, Sansone LA. Antidepressant adherence: are patients taking their medications? Innov Clin Neurosci [Internet]. 2012 [cited 2012 october 29];9(56):41-6. Available from: http://www.ncbi.nlm.nih.gov/ pubmed/22808448

5. Miguel EC, Gentil V, Gattaz WF. Clínica psiquiátrica. Barueri (SP): Manole; 2011.

6. Al-Harbi KS. Treatment-resistant depression: therapeutic trends, challenges, and future directions. Patient Prefer Adherence [Internet]. 2012 [cited 2012 october 29];6:369-88. Available from: http://www.ncbi.nlm.nih. gov/pubmed/22654508

7. Kennedy $\mathrm{SH}$. A review of antidepressant therapy in primary care: current practices and future directions. Prim Care Companion CNS Disord [Internet]. 2013 [cited 2012 october 29];15(2). Available from: http://www.ncbi.nlm. nih.gov/pubmed/23930234

8. Delgado $A B$, Lima ML. Contributo para validação concorrente de uma medida de adesão aos tratamentos. Psicol Saúde Doenças [Internet]. 2001 [acesso em 29 de outubro de 2012];2(2):81-100. Disponível em: http:// www.scielo.oces.mctes.pt/scielo.php?pid = S1645$-00862001000200006 \&$ script $=$ sci_arttext

9. Stape DDB. O conhecimento do paciente com alta hospitalar sobre a continuidade do seu tratamento [dissertação]. São Paulo (SP): Escola de Enfermagem da Universidade de São Paulo; 1979.

10. Minayo MCS. O desafio do conhecimento: pesquisa qualitativa em saúde. 11. ed. São Paulo (SP): Hucitec; 2012.

11. Grenard JL, Munjas BA, Adams JL, Suttorp M, Maglione M, McGlynn EA, Gellad WF. Depression and medication adherence in the treatment of chronic diseases in the United States: a meta-analysis. J Gen Intern Med [Internet].
2011 [cited 2012 october 29];26(10):1175-82. Available from: http://www.ncbi.nlm.nih.gov/pubmed/21533823

12. Párraga Martinez I, López-Torres Hidalgo J, Del Campo Del Campo JM, Villena Ferrer A, Morena Rayo S, Rabadán $\mathrm{E}$, et al. Adherence to patients antidepressant treatment and the factors associated of non-compiance. Aten Primaria [Internet]. 2014 [cited 2012 october 29]:S02126567(13)00308-9. Available from: http://www.ncbi.nlm. nih.gov/pubmed/24704196

13. Burton C, Anderson N, Wilde K, Simpson CR. Factors associated with duration of new antidepressant treatment: analysis of a large primary care database. Br J Gen Pract [Internet]. 2012 [cited 2012 october 29];62(595):e10412. Available from: http://www.ncbi.nlm.nih.gov/ pubmed/22520784

14. Hawton K, Casañas I Comabella C, Haw C, Saunders K. Risk factors for suicide in individuals with depression: a systematic review. J Affect Disord [Internet]. 2013[cited 2012 october 29];147(1-3):17-28. Available from: http:// www.ncbi.nlm.nih.gov/pubmed/23411024

15. Bolkan CR, Bonner LM, Campbell DG, Lanto A, Zivin K, Chaney $\mathrm{E}$, et al. Family involvement, medication adherence, and depression outcomes among patients in veterans affairs primary care. Psychiatr Serv [Internet]. 2013 [cited 2012 october 29]:64(5):472-8. Available from: http://www.ncbi.nlm.nih.gov/pubmed/23370463

16. Cruz LP, Miranda PM, Vedana KGG, Miasso Al. Terapêutica medicamentosa: adesão, conhecimento e dificuldades de idosos com transtorno bipolar. Revista Latinoam Enferm [Internet]. 2011 [acesso em 29 de outubro de 2012];19(4):944-52. Disponível em: http://www.scielo.br/ scielo.php?pid $=$ S0104-11692011000400013\&script $=$ sci arttext\&tlng $=\mathrm{pt}$

17. Monteschi M, Vedana KGG, Miasso Al. Terapêutica medicamentosa: conhecimento e dificuldades de familiares de pessoas idosas com transtorno afetivo bipolar. Texto \& Contexto Enferm [Internet]. 2010 [acesso em 29 de outubro de 2012];19(4):709-18. Disponível em: http://www.scielo.br/scielo.php?script = sci arttext\&pid $=$ S0104-07072010000400014

18. Cavalheri SC. Transformações do modelo assistencial em saúde mental e seu impacto na família. Rev Bras Enferm [Internet]. 2010 [acesso em 29 de outubro de 2012];63(1):51-7. Disponível em: http://www.scielo.br/ scielo.php?pid $=$ S0034-71672010000100009\& script $=$ sci abstract\&tlng $=$ pt

19. Moimaz SAS, Marques JAM, Saliba O, Garbin CAS, Zina LG, Saliba NA. Satisfação e percepção do usuário do SUS sobre o serviço público de saúde. Physis (Rio J.) [Internet]. 2010 [acesso em 29 de outubro de 2012];20(4):1419-40. Disponível em: http://www.scielo.br/scielo.php?pid=S0103-7 3312010000400019\&script $=$ sci arttext 